\title{
Estimation of Xyloglucan in Vegetables by Enzyme-Linked Immunosorbent Assay
}

\author{
Yoshiaki SonE* and Yumiko FuJIKAwA \\ Department of Food and Nutrition, Faculty of Science of Living, \\ Osaka City University, Sumiyoshi-ku, Osaka 558, Japan
}

(Received July 12, 1993)

\begin{abstract}
Summary We prepared the antibody specific against plant xyloglucan and applied it to enzyme-linked immunosorbent assay in order to estimate xyloglucan contents in several vegetables. In this experiment, xyloglucan was detected in the $24 \%$ aqueous potassium hydroxide-soluble fractions of tomato, cabbage, lettuce, and eggplant. The xyloglucan contents of these vegetables were estimated to be $1.4 \mathrm{mg}, 54 \mu \mathrm{g}, 14 \mu \mathrm{g}$, and $4 \mu \mathrm{g}$ per one hundred grams of their edible portions, respectively, using tamarind xyloglucan as a standard xyloglucan.
\end{abstract}

Key Words xyloglucan, hemicellulose, dietary fiber, ELISA

Since Burkitt and Trowell proposed that a lack of fiber-rich foods in the diet may be associated with incidence of certain chronic disorders (1), a lot of reports concerning physical and physiological properties of dietary fibers have been published. In the course of our investigation on the chemical structure of xyloglucan of tamarind seed, recently, we found that the oligosaccharides derived from the xyloglucan inhibit intestinal glucose absorption in rat (2). Because xyloglucans in plant cell wall consist of the repeating units similar to those of tamarind xyloglucan and they occur in plant as one of hemicelluloses (3), this finding suggests a possibility that xyloglucans in vegetables have some physiological effects on animal digestive system, particularly in the small intestine. From this point of view, we designed to analyze xyloglucan in commercially available vegetables.

Usual methods for dietary fiber analysis fall into one of two categories, such as gravimetric and component analyses (4), but both methods cannot estimate the amount of each individual fiber. Since antibodies can have specificity for biological macromolecules, enzyme-linked immunosorbent assay (ELISA) has been developed in the field of biochemical analysis. Concerning the analysis of plant xyloglucan, we have already prepared the antibody specific against the repeating units of tamarind xyloglucan $(5,6)$ and reported that "sandwich type" ELISA could determine not only tamarind xyloglucan but also pea xyloglucan which contains L-fucosyl residues as non-reducing terminal sugars (7). These facts have

\footnotetext{
* To whom reprint requests should be addressed.
} 
prompted us to apply ELISA method to estimate the xyloglucan contents in some vegetables.

In this paper, we describe the fractionation of edible portion of vegetables, the analysis of xyloglucan in each fraction by ELISA using the antibody specific against tamarind xyloglucan, and confirmation of the effectiveness of this ELISA method through detection of xyloglucan molecule by methylation analysis of each fraction.

\section{MATERIALS AND METHODS}

Fractionation of edible portion of vegetables. The vegetables used in this experiment were eggplant, tomato, carrot, radish, onion, cabbage, and lettuce. They were all commercially available in the middle of September at the local market. The extract obtained by each treatment was dialyzed against running water and lyophilized. All centrifugations were done at $10,000 \mathrm{rpm}$ for $30 \mathrm{~min}$ using TOMY centrifuge rotor No. 9N. First, the edible portion of vegetable $(60 \mathrm{~g})$ was immersed in phosphate-buffered saline (PBS, $150 \mathrm{ml}$ ) and homogenized with a blender. The homogeneous suspension was stirred at $4{ }^{\circ} \mathrm{C}$ for $24 \mathrm{~h}$. The soluble fraction (PBS extract) and the insoluble residue were separated by centrifugation. The insoluble residue was suspended in $70 \mathrm{ml}$ of PBS and autoclaved for $20 \mathrm{~min}$. The suspension was centrifuged to give the soluble fraction (hot-water extract). The precipitate was suspended in $50 \mathrm{ml}$ of $4 \%$ aqueous potassium hydroxide containing $0.1 \%$ sodium borohydride $\left(\mathrm{NaBH}_{4}\right)$, stirred at room temperature overnight, and then it was centrifuged. The obtained supernatant $(4 \% \mathrm{KOH}$ extract) was neutralized with acetic acid, and dialyzed against running water. The insoluble residue after $4 \% \mathrm{KOH}$ extraction was then suspended in $24 \%$ aqueous potassium hydroxide $(50 \mathrm{ml})$ containing $0.1 \% \mathrm{NaBH}_{4}$ and stirred at room temperature overnight. The soluble fraction $(24 \% \mathrm{KOH}$ extract) was prepared by the same method as that for $4 \% \mathrm{KOH}$ extract. The insoluble residue after $24 \% \mathrm{KOH}$ extraction was washed thoroughly with distilled water and lyophilized. The fractionation procedure is depicted in Fig. 1.

Preparation of anti-xyloglucan antibody. The anti-xyloglucan serum was prepared by immunization of rabbit with the glycoconjugate of bovine serum albumin (BSA) and the nonasaccharide repeating unit (XGO9) of tamarind xyloglucan as described previously (5). Prior to isolation of immunoglobulin $\mathrm{G}$ (IgG) from the antiserum, the anti-BSA antibody which had been raised against the carrier protein (BSA) was removed by BSA-conjugated Sepharose 4B gel. IgG fraction containing the anti-xyloglucan antibody (anti-XG) was obtained from the antiserum with Affi-Gel Protein A MAPS II kit (Japan BIO-RAD, Tokyo) according to its instruction manual. Alkaline phosphatase-labeled anti-XG (AP-anti-XG) was prepared by the method of Avrameas ( 8 ) using alkaline phosphatase from bovine intestinal mucosa (type VII-T, Sigma) and glutaraldehyde as a cross-linking reagent. Quantitative precipitation and inhibition tests of anti-XG were carried out according to the method of Kabat and Mayer (9). 


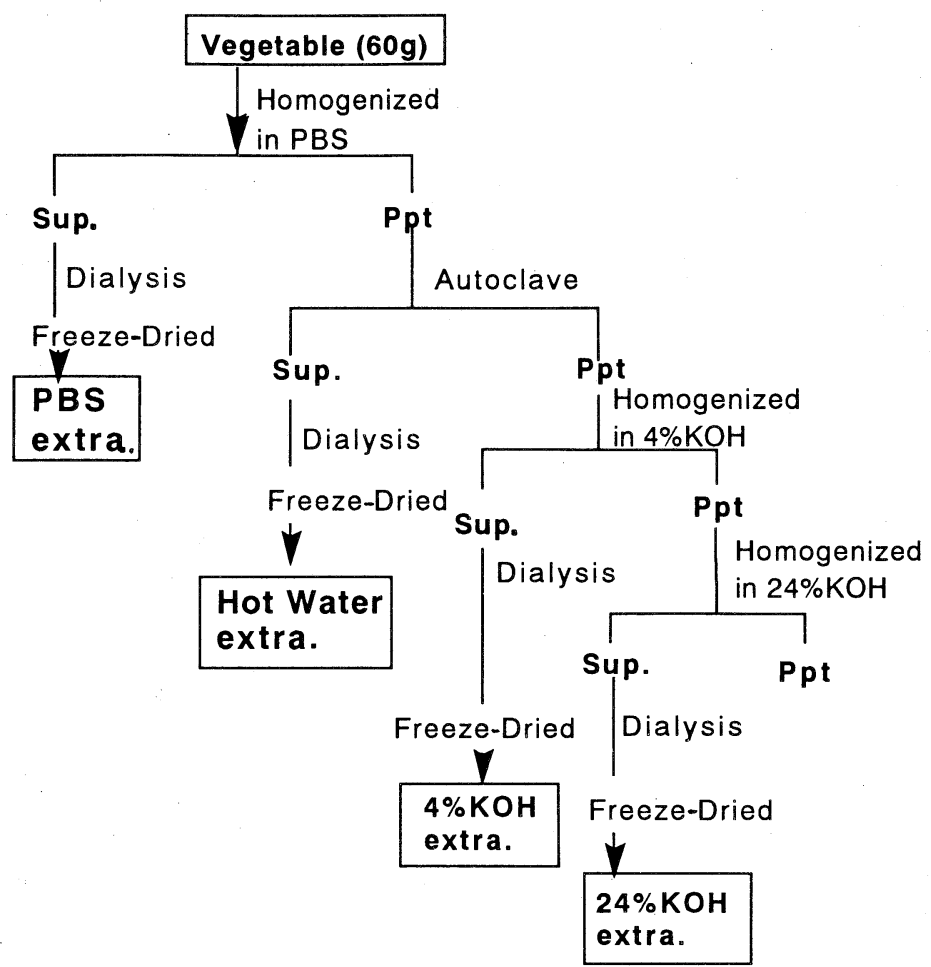

Fig. 1. Fractionation procedure of edible portion of vegetable.

Procedure for xyloglucan analysis by ELISA. ELISA plates (96 well, flat bottom) were purchased from Corning, New York, USA. Each fraction was suspended in PBS and agitated in ultra-sonic bath at $47 \mathrm{kHz}$ for $30 \mathrm{~min}$. The procedure for analysis of xyloglucan in each fraction of the vegetable by ELISA is almost the same as that described in the previous paper (7), but in this experiment 0.02\% Tween 20 in PBS (PBS-Tween) was used as the solvent for AP-anti-XG and diluent for sample solutions (10\% fetal calf serum in PBS was used previously). At the final step of ELISA, the enzyme was allowed to react with the substrate at $37^{\circ} \mathrm{C}$ for $30 \mathrm{~min}$. Absorbance at the well with the addition of PBS-Tween instead of sample solution was referred to as a blank. Tamarind xyloglucan solution $(1 \mu \mathrm{g} / \mathrm{ml}$ PBS) was used to make a standard curve.

Other analytical methods. Methylation of each extract and the following gas liquid chromatography-mass spectrometoric analysis (GLC-mass) of methyl sugars were carried out by the method described by Svensson et al. (10). GLC-mass of methylated sugars was recorded by Hitachi M-2000 mass spectrometer. 


\section{RESULTS}

Figure 2 shows quantitative precipitation curve between tamarind xyloglucan and the antiserum prepared against the conjugate of BSA and XGO9 (BSAXGO9). After removal of the anti-BSA antibody, $100 \mu 1$ of antiserum reacted with $5 \mu \mathrm{g}$ of tamarind xyloglucan to give $38 \mu \mathrm{g}$ of precipitated antibody protein. At the maximum point of precipitation, $0.5 \mu \mathrm{mol}$ of XGO9 inhibited the precipitation by $100 \%$, and at the same conditions, $0.5 \mu \mathrm{mol}$ of XGO8 and XGO7 inhibited the reaction by 33 and $22 \%$, respectively. The purified antibody was coupled with alkaline-phosphatase, and the specificity of the enzyme-labeled antibody (AP-anti-

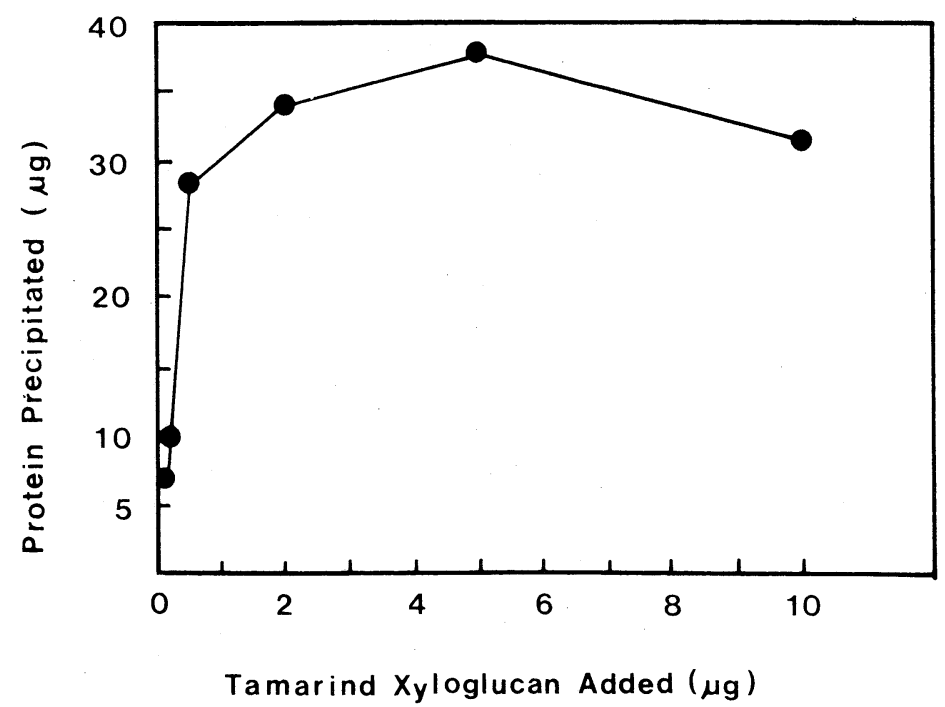

Fig. 2. Quantitative precipitation reaction between tamarind xyloglucan and anti-XGO9 antiserum.

Table 1. Yield of extract (mg).

\begin{tabular}{lrccc}
\hline & PBS & Hot water & $4 \%$ KOH & $24 \% \mathbf{~ K O H}$ \\
\hline Eggplant & 249 & 178 & 159 & 292 \\
Tomato & 113 & 67 & 25 & 107 \\
Cabbage & 97 & 109 & 19 & 213 \\
Onion & 79 & 193 & 52 & 167 \\
Lettuce & 188 & 254 & 50 & 49 \\
Carrot & 164 & 361 & 54 & 72 \\
Radish & 171 & 192 & 41 & 34 \\
\hline
\end{tabular}

Yield from $60 \mathrm{~g}$ of edible portion of vegetable. 
XG) was confirmed by its titration test on ELISA plate using BSA-XGO9 as the immobilized antigen. Prepared AP-anti-XG reacted with BSA-XGO9 but not with BSA, and the reaction was inhibited by the addition of XGO9.

In order to isolate the fraction which contains xyloglucan molecule, the edible portions of the vegetables were fractionated as shown in Fig. 1. Four extracts, such as PBS extract, hot-water extract, 4\% $\mathrm{KOH}$ extract, and $24 \% \mathrm{KOH}$ extract, were obtained in the yields described in Table 1 . These lyophilized extracts were subjected to ELISA and to methylation analysis.

Xyloglucan content of each fraction was estimated by the ELISA method as described in MATERIALS AND METHODS. Each lyophylized extracts were suspended in PBS and agitated in ultrasonic bath before ELISA to make sample solution. All the sample solution contained a small amount of insoluble material even after 30 min ultrasonic treatment, but preliminary experiment showed that it has no effect on xyloglucan determination by this method. Figure 3 shows typical titration curves of $4 \% \mathrm{KOH}$ extracts of onion, eggplant, and cabbage. It is apparent that absorbances at $410 \mathrm{~nm}$ of wells were constant around zero. Titration curves of PBS extracts, hot-water extracts, $4 \% \mathrm{KOH}$ extracts of any vegetables gave the same titration curves as those in Fig. 3 (data not shown). These results suggested that the amounts of xyloglucan in those extracts were too small to be detected by this ELISA method. Figure 4 shows typical titration curves of $24 \% \mathrm{KOH}$ extracts of tomato and lettuce. The curves are almost parallel to that of tamarind xyloglucan,

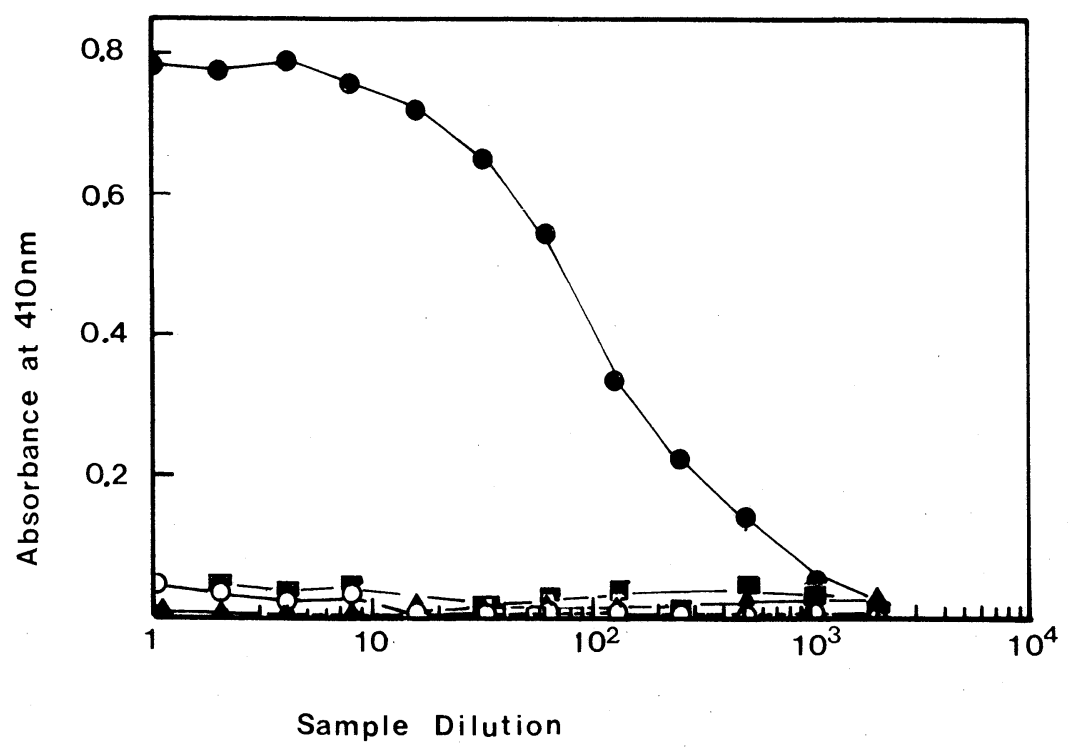

Fig. 3. Assay of xyloglucan in $4 \% \mathrm{KOH}$ extracts by ELISA. $\bullet$, tamarind xyloglucan $(1 \mathrm{mg} / \mathrm{ml}$ at dilution 1$) ; \mathbf{\square}, 4 \% \mathrm{KOH}$ extract of eggplant; $\bigcirc, 4 \%$ $\mathrm{KOH}$ extract of cabbage; $\triangle, 4 \% \mathrm{KOH}$ extract of onion. 


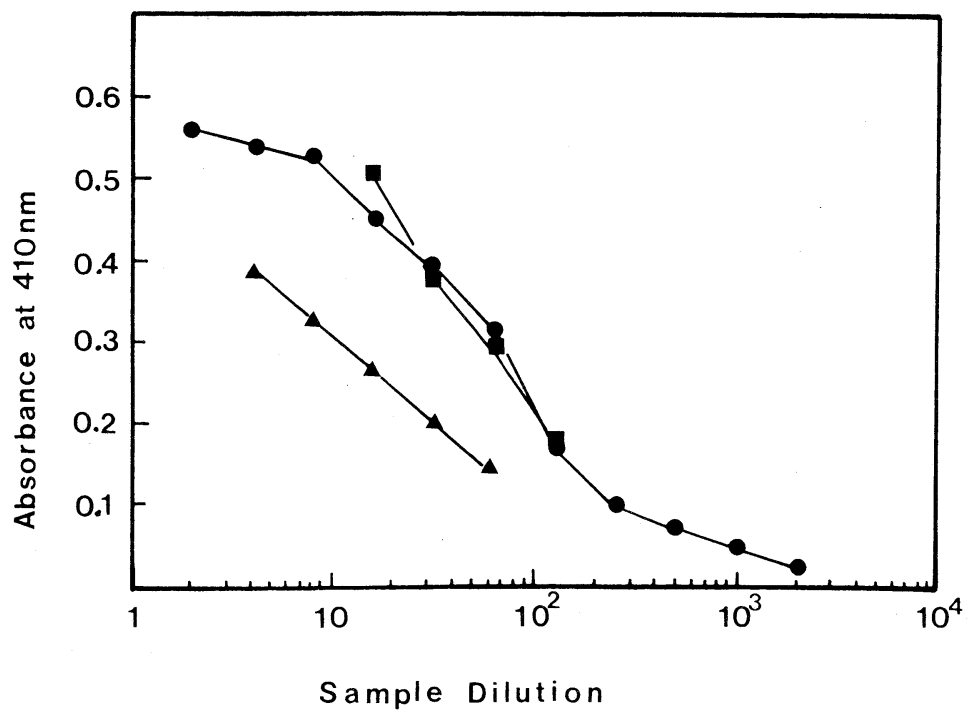

Fig. 4. Assay of xyloglucan in $24 \% \mathrm{KOH}$ extracts by ELISA. @, tamarind xyloglucan $(1 \mathrm{mg} / \mathrm{ml}$ at dilution 1$) ; \boldsymbol{\square}, 24 \% \mathrm{KOH}$ extract of tomato; $\mathbf{\Delta}, 24 \%$ $\mathrm{KOH}$ extract of lettuce.

so the content of xyloglucan in each sample solution can be calculated by the comparison to that of standard tamarind xyloglucan on consideration of the dilution factor of the sample solution. On the other hand, titration curves of the $24 \% \mathrm{KOH}$ extracts of carrot, radish, and onion, were the same as those in Fig. 3 (data not shown).

The presence of xyloglucan in each fraction was confirmed by methylation analysis. Each extract was methylated and methyl sugars in its hydrolyzate were analyzed by GLC-mass. Figure 5 shows the GLC pattern of the acid hydrolyzate of methylated $24 \% \mathrm{KOH}$ extract of tomato. These main peaks were identified to be 3,4-di-O-methyl 1,2,5-tri-O-acetyl D-xylitol (I), 2,3,4,6-tetra-O-methyl 1,5-di-Oacetyl D-galactitol (II), 2,3,6-tri-O-methyl 1,4,5-tri-O-acetyl D-glucitol (III), and 2,3-di-O-methyl 1,4,5,6-tetra-O-acetyl D-glucitol (IV) by their mass chromatographic pattern. This GLC pattern is almost the same as that of methylated tamarind xyloglucan except that 2,3,4-tri-O-methyl 1,5-di-O-acetyl D-xylitol was not detected. Methylated extracts in which no xyloglucan was detected by ELISA did not contain methylated sugars such as those detected in methylated xyloglucan (data not shown). The residues after $24 \%$ alkaline treatment of all vegetables gave no methyl sugars from plant xyloglucan on their methylation analyses (data not shown). 


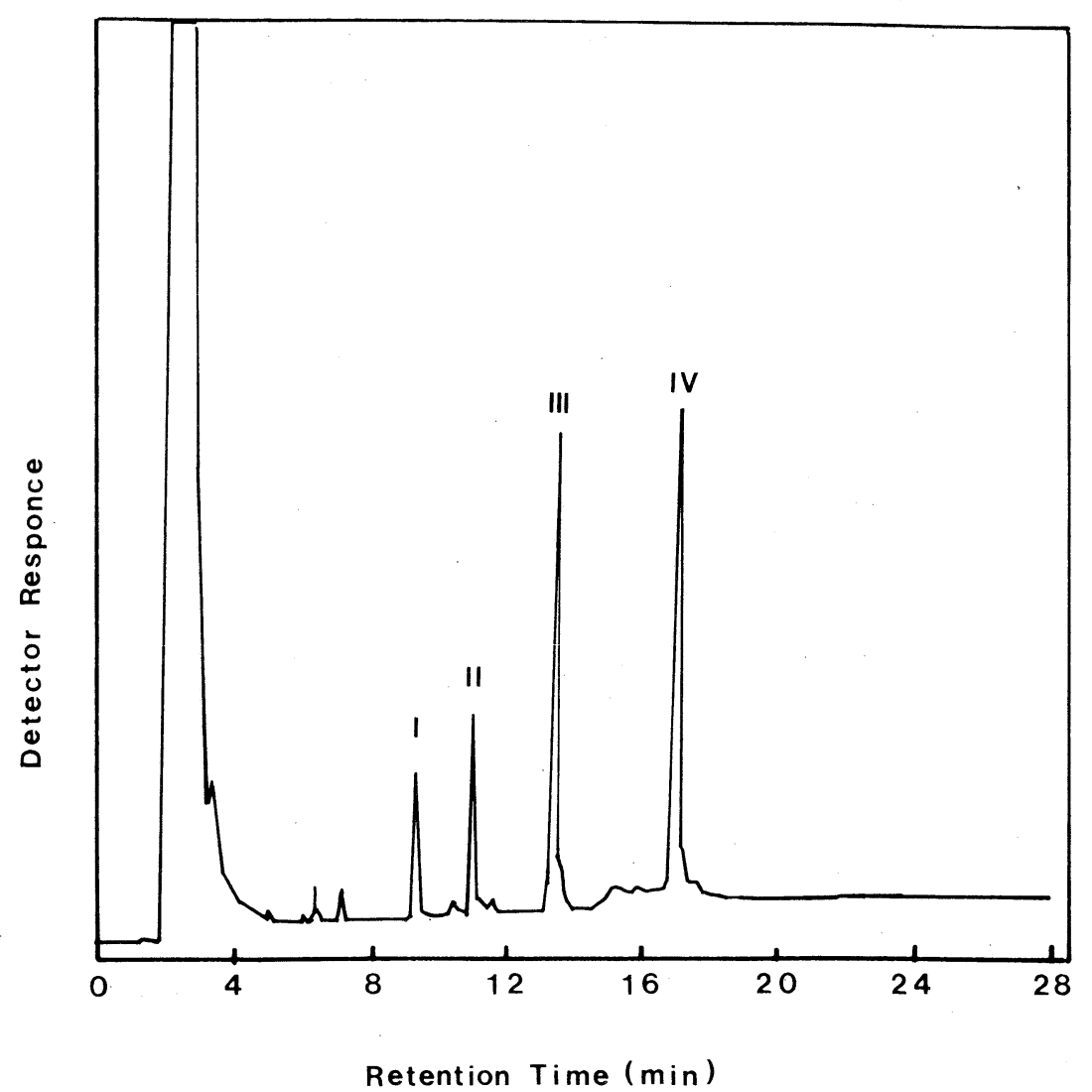

Fig. 5. GLC pattern of hydrolyzate of methylated $24 \% \mathrm{KOH}$ extract of tomato. I: 3,4 -di-O-methyl xylose; II: 2,3,4,6 -tetra-O-methyl galactose; III: 2,3,6-tri-Omethyl glucose; IV: 2,3-di-O-methyl glucose.

\section{DISCUSSION}

Progress in understanding the action of different types of dietary fiber in the intestinal tract shows that each plant cell wall polysaccharide could be expected to have proper physiological effect due to their physical properties. For example, pectic polysaccharides have a mineral binding property through their cation exchange capacity (4). Recently, we found that the oligosaccharides obtained from tamarind xyloglucan, of which chemical structure is almost the same as those in plant cell wall, have inhibitory effects on absorption of D-glucose in rat small intestine probably because they act as competitive inhibitors on glucose binding sites (2). These facts demand a special analytical method to a certain cell wall polysaccharide. Since ELISA method is suitable for this purpose, we applied it to estimate the xyloglucan contents in commercially available vegetables. 
In ELISA method, it is important to identify the specificity of the antibody used. From the results of oligosaccharides-inhibition of the precipitation of the antibody by tamarind xyloglucan, it is apparent that the antibody used in this experiment is specific to nonasaccharide repeating unit of tamarind xyloglucan. The nonasaccharide unit is a common unit to plant xyloglucan because that cell-wall xyloglucans possess a $1,4-\beta$-glucan backbone with $\alpha$-xylosyl residues attached to O- 6 of $\beta$-D-glucosyl residues, and the terminal galactosyl residues are bound to O-2 of D-xylosyl residues by $\beta$-linkage (11). Therefore, this antibody is able to be applied for ELISA determination of plant xyloglucan. Fucosylated xyloglucan occurs in the cell wall of dicotyledon (11); however, previous experiment showed that the presence of fucosyl residues on D-galactosyl residues has no effect on this ELISA method (7).

Concerning the localization of xyloglucans in plant cell wall, Hayashi (11) has reported that they are firmly associated with cellulose microfibril, so they are extracted only by strong alkali solution $(24 \%$ aqueous $\mathrm{KOH})$ but not either by hot-water or by weak alkali (4\% aqueous $\mathrm{KOH})$. According to Hayashi's report and the fractionation procedure used in our previous experiments concerning the structural studies of polysaccharides of edible mushrooms $(12,13)$, edible portion of vegetable was fractionated as shown in Fig. 1. After extraction of water-soluble materials by homogenization in PBS and by autoclave, xyloglucan should be extracted in the $24 \% \mathrm{KOH}$ extract.

Xyloglucan content in each extract was determined by ELISA method. In twenty-eight extracts tested, xyloglucan was detected in four $24 \% \mathrm{KOH}$ extracts, such as those from tomato, cabbage, lettuce, and eggplant. Titrations of the other extracts showed that they don't contain detectable amount of the xyloglucan. As shown in Fig. 4, the titration curve of $24 \% \mathrm{KOH}$ extract of tomato is parallel to that of tamarind xyloglucan in the range of sample dilution between $1: 20$ and $1: 100$. Table 2 summarizes the total xyloglucan contents calculated from the titration curves on consideration of the dilution factor of the sample solution.

Methylation analyses of the extracts were carried out to confirm the presence of xyloglucan in the $24 \% \mathrm{KOH}$ extracts of tomato, cabbage, lettuce, and eggplant, in which the xyloglucan was detected by ELISA. From the structural feature of xyloglucan and other polysaccharides which occur in plant cell wall (3), 3,4-

Table 2. Estimated xyloglucan content.

\begin{tabular}{lc}
\hline & Xyloglucan $(\mu \mathrm{g})^{1}$ \\
\hline Tomato & 1,370 \\
Cabbage & 54 \\
Lettuce & 14 \\
Eggplant & 4 \\
\hline
\end{tabular}

${ }^{1}$ Content equivalent to the amounts of tamarind xyloglucan in $100 \mathrm{~g}$ of edible portion of vegetable. 
di-O-methyl xylose (derived from the 2-linked xylosyl residue) and 2,3-di-O-methyl glucose (derived from the 4,6-linked backbone glucosyl residue) should be included in the hydrolyzate of methylated extracts which contain the xyloglucan molecule. Besides the methyl sugars mentioned above, 2,3,4,6-tetra-O-methyl galactose (from non-reducing galactose terminal), 2,3,4-tri-O-methyl xylose (from non-reducing xylose terminal), 2,3,4-tri-O-methyl L-fucose (from non-reducing fucose terminal), and 2,3,6-tri-O-methyl glucose (from the 4-linked D-glucose backbone) could be derived from not only the xyloglucan but also other polysaccharides in cell wall. As shown in Fig. 5, the acid hydrolyzate of methylated 24\% $\mathrm{KOH}$ extract of tomato contained the methyl sugars characteristic to methylated plant xyloglucan. Methylated $24 \% \mathrm{KOH}$ extracts of eggplant, cabbage, and lettuce gave almost the same GLC patterns as that of tomato. These facts clearly showed the presence of xyloglucan in these extracts. On the other hand, acid hydrolyzates of methylated $24 \% \mathrm{KOH}$ extracts in which no xyloglucan was detected by ELISA (of onion, radish, and carrot) gave no methyl sugars characteristic to the xyloglucan, when the same quantity of the hydrolyzate as that of $24 \% \mathrm{KOH}$ tomato extract was analyzed by GLC-mass. In addition, methylation analysis of the $24 \% \mathrm{KOH}$ insoluble residues of all vegetables showed that they contain no xyloglucan. These results confirm that the xyloglucan in the vegetable was extracted in $24 \% \mathrm{KOH}$ extracts, if present, and that this ELISA method is effective to estimate the xyloglucan content in vegetables.

\section{REFERENCES}

1) Burkitt, D. P., and Trowell, H. (1975): Refined Carbohydrate Foods and Disease, Academic Press, New York.

2) Sone, Y., Makino, C., and Misaki, A. (1992): Inhibitory effect of oligosaccharides derived from plant xyloglucan on intestinal glucose absorption in rat. J. Nutr. Sci. Vitaminol., 38, 391-395.

3) Prakash, M. D., and Brinson, K. (1987): Plant cell-wall. Adv. Carbohydr. Chem. Biochem., 42, 265-382.

4) Schneeman, B. O., and Gallaher, D. D. (1990): Dietary fiber, in Present Knowledge in Nutrition, 6th Ed., ed. by Brown, M. L., International Life Sciences Institute Nutrition Foundation, Washington, D.C., pp. 80-87.

5) Sone, Y., Kuramae, J., Shibata, S., and Misaki, A. (1989): Immunochemical specificities of antibody to the heptasaccharide unit of plant xyloglucan. Agric. Biol. Chem., 53, 2821-2823.

6) Sone, Y., Misaki, A., and Shibata, S. (1989): Preparation and characterization of antibodies against 6-O- $\alpha$-D-xylopyranosyl- $\beta$-D-glucopyranose ( $\beta$-isoprimeverose), the disaccharide unit of xyloglucan in plant cell-walls. Carbohydr. Res., 191, 79-89.

7) Sone, Y., Sato, K., and Misaki, A. (1991): Determination of plant xyloglucan by enzyme-linked immunosorbent assay. Agric. Biol. Chem., 55, 2155-2156.

8) Avrameas, S. (1969): Coupling of enzymes to proteins with glutaraldehyde. Use of the conjugate for the detection of antigens and antibodies. Immunochemistry, 6, 43-52. 
9) Kabat, E. A., and Mayer, M. M. (1971): Immunological and immunochemical methodology, in Kobat and Mayer's Experimental Immunochemistry, Charles C Thomas, Springfield, Illinois, pp. 3-326.

10) Bjorndal, H., Hellerqvist, C. G., Lindberg, B., and Svensson, S. (1970): Gas-liquid chromatography and mass spectrometry in methylation analysis of polysaccharides. Angew. Chem. Int. Ed., 9, 610-619.

11) Hayashi, T. (1989): Xyloglucan in the primary cell wall. Annu. Rev. Plant Physiol. Plant Mol. Biol., 40, 139-168.

12) Sone, Y., Kakuta, M., and Misaki, A. (1978): Isolation and characterization of polysaccharides of "Kukurage," fruit body of Auricularia auricula-judae. Agric. Biol. Chem., 42, 417-425.

13) Misaki, A., Nasu, M., Sone, Y., Kishida, E., and Kinoshita, C. (1986): Comparison of structure and antitumor activity of polysaccharides isolated from Fukurotake, the fruiting body of Volvariella volvacea. Agric. Biol. Chem., 50, 2171-2183. 